\title{
The Langberg-Médard Multiple Unicast Conjecture: Stable 3-Pair Networks *
}

\author{
Kai Cai \\ Guangyue Han \\ The University of Hong Kong The University of Hong Kong \\ email: kcai@hku.hk email: ghan@hku.hk
}

April 21, 2020

\begin{abstract}
The Langberg-Médard multiple unicast conjecture claims that for a strongly reachable $k$-pair network, there exists a multi-flow with rate $(1,1, \ldots, 1)$. In this paper, we show that the conjecture holds true for stable 3-pair networks.
\end{abstract}

\section{Introduction}

A $k$-pair network $\mathcal{N}=(V, A, S, T)$ consists of a directed acyclic graph (DAG) $D=(V, A)$, a set of $k$ vertices $S=\left\{s_{1}, s_{2}, \ldots, s_{k}\right\}$ with zero in-degree, called sources (senders) and a set of $k$ vertices $T=\left\{t_{1}, t_{2}, \ldots, t_{k}\right\}$ with zero out-degree, called sinks (receivers). For convenience only, we assume that any $k$-pair network considered in this paper does not have vertices whose in-degree and out-degree are both equal to 1. Roughly put, the multiple unicast conjecture, also known as the $L i$ - $L i$ conjecture [11, claims that for any $k$-pair network, if information can be transmitted from all the senders to their corresponding receivers at rate $\left(r_{1}, r_{2}, \ldots, r_{k}\right)$ via network coding, then it can be transmitted at the same rate via undirected fractional routing. One of the most challenging problems in the theory of network coding [16], this conjecture has been doggedly resisting a series of attacks [1, 2, 3, 4, 17, 8, 10, 12, 14, 15] and is still open to date.

A $k$-pair network $\mathcal{N}$ is said to be fully reachable if there exists an $s_{i}$ - $r_{j}$ directed path $P_{s_{i}, r_{j}}$ for all $i, j$; and strongly reachable if, in addition, the paths $P_{s_{1}, r_{j}}, P_{s_{2}, r_{j}}, \cdots, P_{s_{k}, r_{j}}$ are edge-disjoint for any $j$; and extra strongly reachable if, furthermore, for any $j$ and all $i \neq k$, $P_{s_{i}, t_{j}}$ and $P_{s_{k}, t_{j}}$ do not share any vertex other than $t_{j}$. Throughout the paper, we will reserve the notations $\mathbf{P}_{t_{j}}$ and $\mathbf{P}$ and define

$$
\mathbf{P}_{t_{j}}:=\left\{P_{s_{i}, t_{j}}: i=1,2, \ldots, k\right\}, \quad \mathbf{P}:=\cup_{j=1}^{k} \mathbf{P}_{t_{j}} .
$$

*This research is partly supported by a grant from the Research Grants Council of the Hong Kong Special Administrative Region, China (Project No. 17301017) and a grant by the National Natural Science Foundation of China (Project No. 61871343). 
For notational convenience, we may refer to a path from $\mathbf{P}_{t_{j}}$ as a $\mathbf{P}_{t_{j}}$-path, or simply a $\mathbf{P}$-path, and moreover, an arc on the path $\mathbf{P}_{t_{j}}$ as a $\mathbf{P}_{t_{j}}$-arc. Note that an arc can be simultaneously a $\mathbf{P}_{t_{j}}$-arc and a $\mathbf{P}_{t_{j^{\prime}}}$-arc, $j \neq j^{\prime}$.

The following Langberg-Médard multiple unicast conjecture [10], which deals with strongly reachable $k$-pair networks, is a weaker version of the Li-Li conjecture. Note that for a strongly reachable network, each source is able to multicast at rate 1 to all the receivers, e.g., the classic butterfly network of two-unicast is such a case.

Conjecture 1.1. For any strongly reachable $k$-pair network, there exists a feasible undirected fractional multi-flow with rate $(1,1, \ldots, 1)$.

It turns out that Conjecture 1.1 is equivalent to the following conjecture, with "strongly reachable" replaced by "extra strongly reachable".

Conjecture 1.2. For any extra strongly reachable $k$-pair network, there exists a feasible undirected fractional multi-flow with rate $(1,1, \ldots, 1)$.

To see the equivalence, note that Conjecture 1.1 trivially implies Conjecture 1.2, and the reverse direction follows from the fact that a strongly reachable $k$-pair network can be transformed to an extra strongly reachable $k$-pair network with a feasible undirected fractional multi-flow mapped to one with the same rate.

The Langberg-Médard multiple unicast conjecture was first proposed in 2009 [10]. In the same paper, the authors constructed a feasible undirected fractional multi-flow with rate $(1 / 3,1 / 3, \ldots, 1 / 3)$ for a strongly reachable $k$-pair network. Recently, we have improved $1 / 3$ to $8 / 9$ for a generic $k$ in [1] and to $11 / 12$ for $k=3,4$ in [4].

A strongly reachable $k$-pair network $\mathcal{N}$ is said to be stable if the choice of each $P_{s_{i}, t_{j}}$, $i, j=1,2, \ldots, k$, is unique, and unstable otherwise (see Fig. 1); here we remark that $\mathcal{N}$ is stable only if it is extra strongly reachable. In this paper, we will establish Conjecture 1.1 for stable 3-pair networks by establishing Conjecture 1.2 for the same family of networks. Our treatment is based on classification of stable 3-pair networks according to their network topologies. Related work on topological analysis of strongly reachable networks can be found in [9] and [6].

The rest of paper is organized as follows. In Section 2, we introduce some basic notions, facts and related tools. In Section 3, we characterize stable $k$-pair networks and subsequently show that there exists an efficient algorithm to determine the stability of a given $k$-pair network. In Section4, we investigate the topological structure of stable 3-pair networks, for which we settle the Langberg-Médard conjecture in Section 5. Finally, the paper is concluded in Section 6.

\section{Preliminaries}

Throughout this section, we consider a fully reachable $k$-pair network $\mathcal{N}$ and adopt all the related notations defined in Section 1 . 


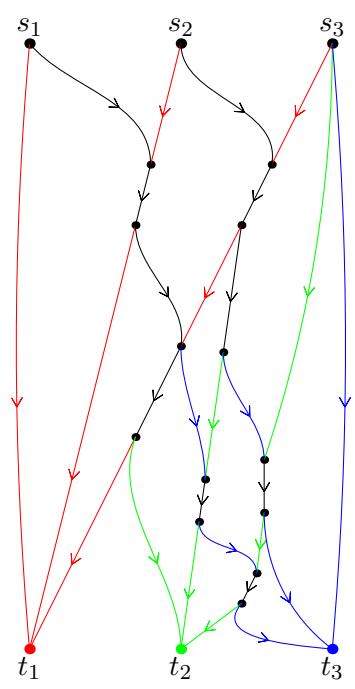

(a)

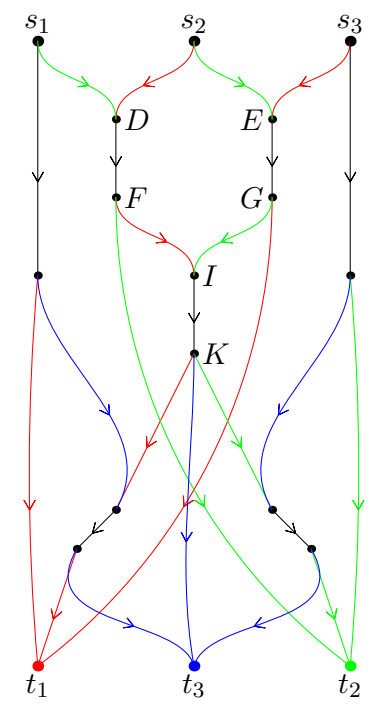

(b)

Figure 1: A stable network (a) and an unstable network (b). In (b), $\mathbf{P}_{t_{2}}$ is not unique since $P_{s_{2}, t_{3}}$ can be chosen as either $\left[s_{2}, D, F, I, K, t_{3}\right]$ or $\left[s_{2}, E, G, I, K, t_{3}\right]$. Here and hereafter, for stable networks, an arc that belongs to only one P-path is colored red, green or blue, respectively, depending on the fact that the $\mathrm{P}$-path is a $\mathbf{P}_{t_{1}}-, \mathbf{P}_{t_{2}}-$ or $\mathbf{P}_{t_{3}}$-path; and an arc that belongs to two or more $\mathbf{P}$-paths' is colored black.

\subsection{Undirected Fractional Multi-Flow}

For an arc $a=[u, v] \in A$, we call $u$ and $v$ the tail and the head of $a$ and denote them by $\operatorname{tail}(a)$, head $(a)$, respectively. For any $s, t \in V$, an $s$ - $t$ flow 1 is a function $f: A \rightarrow \mathbb{R}$ satisfying the following flow conservation law: for any $v \notin\{s, t\}$,

$$
\operatorname{excess}_{f}(v)=0
$$

where

$$
\operatorname{excess}_{f}(v):=\sum_{a \in A: \operatorname{head}(a)=v} f(a)-\sum_{a \in A: \operatorname{tail}(a)=v} f(a) .
$$

It is easy to see that $\operatorname{excess}_{f}(s)=-\operatorname{excess}_{f}(t)$, which is called the value (or rate) of $f$. We say $f$ is feasible if $|f(a)| \leq 1$ for all $a \in A$.

An $\left(s_{1}, s_{2}, \ldots, s_{k}\right)-\left(t_{1}, t_{2}, \ldots, t_{k}\right)$ multi-flow refers to a set of $k$ flows $\mathcal{F}=\left\{f_{1}, f_{2}, \ldots, f_{k}\right\}$, where each $f_{i}$ is an $s_{i}$ - $t_{i}$ flow. We say $\mathcal{F}$ has rate $\left(d_{1}, d_{2}, \ldots, d_{k}\right)$, where $d_{i}:=\operatorname{excess}_{f_{i}}\left(s_{i}\right)$. For any given $a \in A$, we define $|\mathcal{F}|(a)$ as

$$
|\mathcal{F}|(a):=\sum_{1 \leq i \leq k}\left|f_{i}(a)\right|
$$

And we say $\mathcal{F}$ is feasible if $|\mathcal{F}|(a) \leq 1$ for all $a \in A$.

\footnotetext{
${ }^{1}$ The flow or multi-flow defined for directed graph in this paper, which can be negative, is equivalent to the flow defined in [13] for undirected graphs, which has to be non-negative.
} 


\subsection{Routing Solution}

For each $P_{s_{i}, t_{j}}$, we define an $s_{i}-t_{j}$ flow $f_{i, j}$ as follows:

$$
f_{i, j}(a)= \begin{cases}1, & a \in P_{s_{i}, t_{j}} \\ 0, & \text { otherwise. }\end{cases}
$$

Definition 2.1. [Linear Routing Solution] An $\left(s_{1}, s_{2}, \ldots, s_{k}\right)-\left(t_{1}, t_{2}, \ldots, t_{k}\right)$ multi-flow $\mathcal{F}=$ $\left\{f_{1}, f_{2}, \ldots, f_{k}\right\}$ is said to be a routing solution for $\mathcal{N}$ if it is feasible with rate $(1,1, \ldots, 1)$. A routing solution is called linear (with respect to $\mathbf{P}$ ), if, for each feasible $l$,

$$
f_{l}=\sum_{i, j=1}^{k} c_{i, j}^{(l)} f_{i, j}
$$

where all $c_{i, j}^{(l)} \in \mathbb{R}$, in which case the solution $\mathcal{F}$ can be equivalently represented by its matrix form $\mathcal{C}=\left(\left(c_{i, j}^{(1)}\right),\left(c_{i, j}^{(2)}\right), \ldots,\left(c_{i, j}^{(k)}\right)\right)$; otherwise, it is called non-linear.

The following theorem [4] is somewhat straightforward.

Theorem 2.2. An $\left(s_{1}, s_{2}, \ldots, s_{k}\right)$ - $\left(t_{1}, t_{2}, \ldots, t_{k}\right)$ multi-flow $\mathcal{F}=\left\{f_{1}, f_{2}, \ldots, f_{k}\right\}$ satisfying (4) has rate $(1,1, \ldots, 1)$ if and only if all $c_{i, j}^{(l)}$ satisfy

$$
\sum_{j=1}^{k} c_{i, j}^{(l)}=0, \text { for all } i \neq l, \quad \sum_{i=1}^{k} c_{i, j}^{(l)}=0, \text { for all } j \neq l, \quad \sum_{i=1}^{k} \sum_{j=1}^{k} c_{i, j}^{(l)}=1, \text { for all } l .
$$
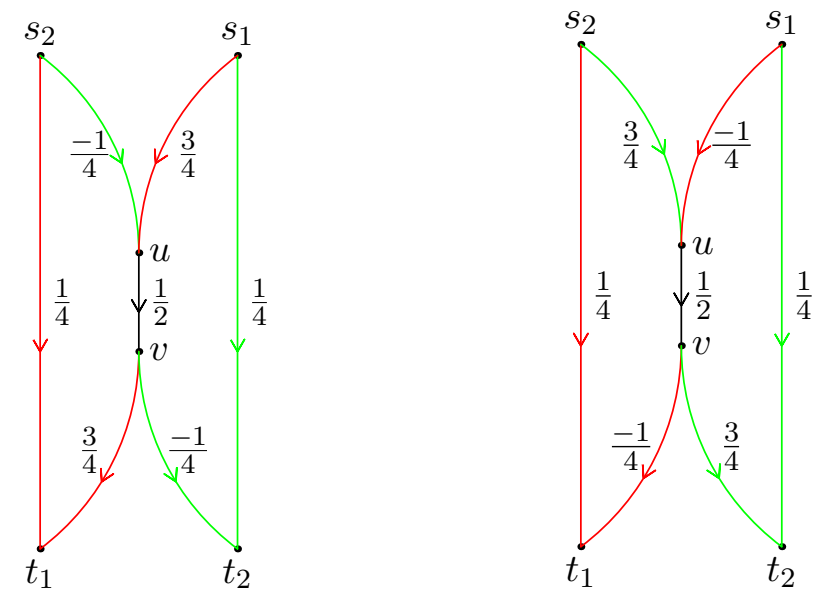

(a) : The $s_{1}-t_{1}$ flow $f_{1}$

(b) : The $s_{2}-t_{2}$ flow $f_{2}$

Figure 2: A Linear routing solution.

Example 2.3. Consider the 2-pair network depicted in Fig. 2 and Fig. 3. It is easy to check that Fig. 2 gives a linear routing solution $\mathcal{F}=\left(f_{1}, f_{2}\right)$ with the matrix form

$$
\left(\left(\begin{array}{cc}
\frac{3}{4} & \frac{1}{4} \\
\frac{1}{4} & \frac{-1}{4}
\end{array}\right),\left(\begin{array}{cc}
\frac{-1}{4} & \frac{1}{4} \\
\frac{1}{4} & \frac{3}{4}
\end{array}\right)\right)
$$


i.e., $f_{1}=\frac{3}{4} f_{1,1}+\frac{1}{4} f_{1,2}-\frac{1}{4} f_{2,2}+\frac{1}{4} f_{2,1}$ and $f_{2}=\frac{3}{4} f_{2,2}+\frac{1}{4} f_{2,1}-\frac{1}{4} f_{1,1}+\frac{1}{4} f_{1,2}$. Note that

$$
|\mathcal{F}|(a)=\left|f_{1}(a)\right|+\left|f_{2}(a)\right|= \begin{cases}\frac{1}{2}, & a \in\left\{\left[s_{1}, t_{2}\right],\left[s_{2}, t_{1}\right]\right\} \\ 1, & \text { otherwise. }\end{cases}
$$

On the other hand, it easy to check that Fig. 3 gives a non-linear routing solution $\mathcal{F}=\left(f_{1}, f_{2}\right)$ with

$$
|\mathcal{F}|(a)= \begin{cases}0, & a=\left[s_{2}, t_{1}\right] \\ 1, & \text { otherwise }\end{cases}
$$
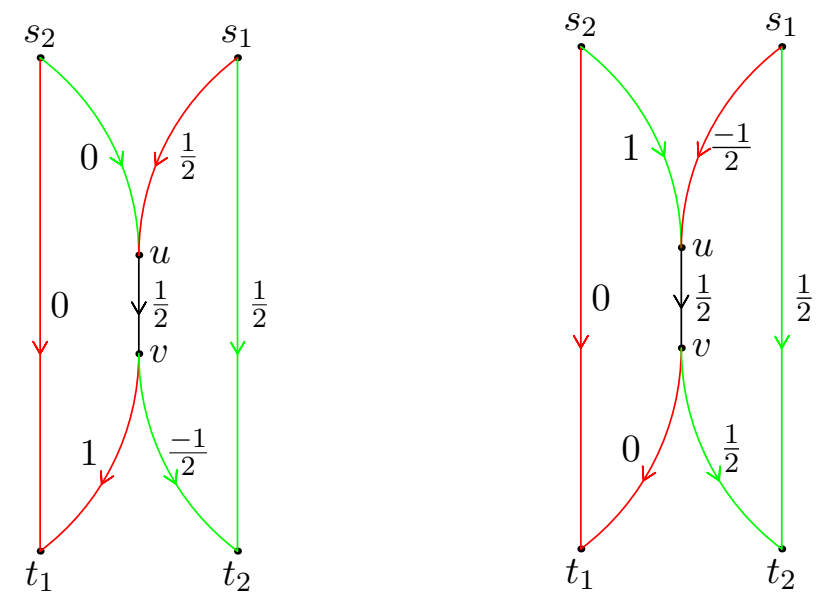

(a) : The $s_{1}-t_{1}$ flow $f_{1}$

(b) : The $s_{2}-t_{2}$ flow $f_{2}$

Figure 3: A non-linear routing solution.

Using the above language, the Langberg-Médard multiple unicast conjecture says that strongly reachable $k$-pair networks always have routing solutions. Here, we conjecture that it can be further strengthened as follows.

Conjecture 2.4. Each strongly reachable k-pair network has a linear routing solution.

\section{$2.3 \mathcal{S}_{\mathcal{N}}$ and $g_{s}(\mathcal{C})$}

Let $[k]=\{1,2, \ldots, k\}$ and define

$$
\mathcal{S}_{\mathcal{N}}=\left\{\left\{(i, j) \in[k] \times[k]: P_{s_{i}, t_{j}} \text { passes through } a\right\}: a \in A\right\} ;
$$

in other words, each element of $\mathcal{S}_{\mathcal{N}}$ is a set of index pairs corresponding to all $\mathbf{P}$-paths that pass through a given arc in $\mathcal{N}$. Note that, if $\mathcal{N}$ is a strongly reachable network, then for any feasible $j$, each arc is passed by at most one of the paths $P_{s_{1}, t_{j}}, P_{s_{2}, t_{j}}, \ldots, P_{s_{k}, t_{j}}$, and hence $\mathcal{S}_{\mathcal{N}} \subseteq \mathcal{S}_{k}$, where

$$
\mathcal{S}_{k}:=\left\{\left\{\left(i_{1}, j_{1}\right), \ldots\left(i_{r}, j_{r}\right)\right\} \subseteq[k] \times[k] \| j_{1}<j_{2}<\cdots<j_{r}, 1 \leq r \leq k\right\} .
$$

Now, for a tuple of $k \times k$ matrices $\mathcal{C}=\left(\left(c_{i, j}^{(1)}\right),\left(c_{i, j}^{(2)}\right), \ldots,\left(c_{i, j}^{(k)}\right)\right)$ satisfying (5) $)$, given $s \in \mathcal{S}_{\mathcal{N}}$ and $l \in[k]$, we define

$$
g_{s}^{(l)}(\mathcal{C}):=\sum_{(i, j) \in s} c_{i, j}^{(l)}
$$


and furthermore,

$$
g_{s}(\mathcal{C}):=\sum_{l=1}^{k}\left|g_{s}^{(l)}(\mathcal{C})\right| .
$$

The following theorem, whose proof is straightforward and thus omitted, will be used as a key tool to establish our results.

Theorem 2.5. $\mathcal{C}$ is a linear routing solution of $\mathcal{N}$ if $g_{s}(\mathcal{C}) \leq 1$ for any $s \in \mathcal{S}_{\mathcal{N}}$.

For $s=\left\{\left(i_{1}, j_{1}\right),\left(i_{2}, j_{2}\right), \ldots,\left(i_{\alpha(s)}, j_{\alpha(s)}\right)\right\} \in \mathcal{S}_{\mathcal{N}}$, we define the following multi-set:

$$
\operatorname{Ind}_{s}:=\left\{i_{1}, j_{1}, i_{2}, j_{2}, \ldots, i_{\alpha(s)}, j_{\alpha(s)}\right\}
$$

where $\alpha(s)$ denotes the size of $s$. And for any $l=1,2, \ldots, k$, denote by $m_{\text {Inds }}(l)$ the multiplicity of $l$ in $I n d_{s}$ (if $l \notin I n d_{s}$, then $m_{I n d_{s}}(l)=0$ ). An element $(i, j) \in s$ is said to be diagonal if $i=j$, otherwise non-diagonal. We use $\gamma(s)$ to denote the number of diagonal elements in $s$. For a quick example, consider $s=\{(1,1),(2,2),(1,3),(3,4),(1,6)\} \subseteq[6] \times[6]$. Then, $\operatorname{Ind}_{s}=\{1,1,2,2,1,3,3,4,1,6\}, m_{\text {Ind }}(1)=4, m_{I n d_{s}}(2)=m_{\text {Ind }}(3)=2, m_{\text {Inds }}(4)=$ $m_{\text {Inds }_{s}}(6)=1, m_{\text {Inds }_{s}}(5)=0, \alpha(s)=5$ and $\gamma(s)=2$.

\section{Characterization of Stable Networks}

In this section, unless specified otherwise, we assume that $\mathcal{N}$ is an extra strongly reachable $k$-pair network.

Definition 3.1. [Residual Network [9] For $j=1,2, \ldots, k$, the $j$-th residual network $\mathcal{N}_{j}$ is formed from $\mathcal{N}$ by reversing the directions of all its $\mathbf{P}_{t_{j}}$-arcs (that may be simultaneously $\mathbf{P}_{t_{j^{\prime}}}$-arcs for some $j^{\prime} \neq j$ ).

Note that in spite of the acyclicity of $\mathcal{N}$, there may exist directed cycles in $\mathcal{N}_{j}$, and such a directed cycle must contain at least one reversed $\mathbf{P}_{t_{j}}$-arc.

Definition 3.2. [Regular Cycle] A directed cycle $C$ of $\mathcal{N}_{j}$ is called regular, if $C$ has no isolated vertex of $\mathbf{P}_{t_{j}}$, otherwise it is called singular.

Definition 3.3. [Semi-Cycle [6]] A $\mathbf{P}_{t_{j}}$-semi-cycle of $\mathcal{N}$ is formed from a regular cycle of $\mathcal{N}_{j}$ by reversing the directions of all its $\mathbf{P}_{t_{j}}$-arcs.

Obviously, there is a one-to-one correspondence from the set of all the $\mathbf{P}_{t_{j}}$-semi-cycles in $\mathcal{N}$ to the set of all the regular cycles of the $j$-th residual network $\mathcal{N}_{j}$.

Definition 3.4. [Crossing] A $\mathbf{P}_{t_{j}}$-crossing of $\mathcal{N}$ is formed from a $\mathbf{P}_{t_{j}}$-semi-cycle of $\mathcal{N}$ by removing all the $\mathbf{P}_{t_{j}}$-arcs.

For example, consider the network $\mathcal{N}$ depicted in (b) of Fig. 1. While the choices of $P_{s_{1}, t_{3}}$ and $P_{s_{3}, t_{3}}$ are both unique, there are two choices for $P_{s_{2}, t_{3}}: P_{s_{2}, t_{3}}^{(1)}=\left[s_{2}, D, F, I, K, t_{3}\right]$ and $P_{s_{2}, t_{3}}^{(2)}=\left[s_{2}, E, G, I, K, t_{3}\right]$, which give rise to two choices of $\mathbf{P}_{t_{3}}: \mathbf{P}_{t_{3}}^{(1)}=\left\{P_{s_{1}, t_{3}}, P_{s_{2}, t_{3}}^{(1)}, P_{s_{3}, t_{3}}\right\}$ 


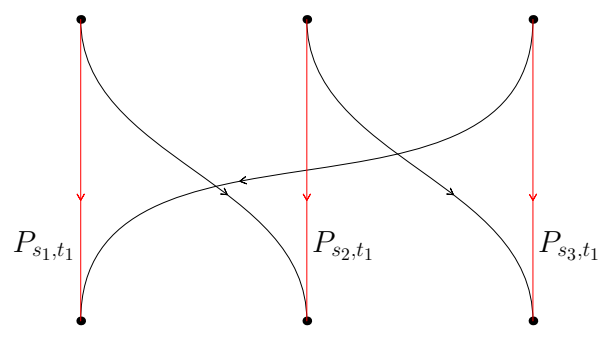

(a)

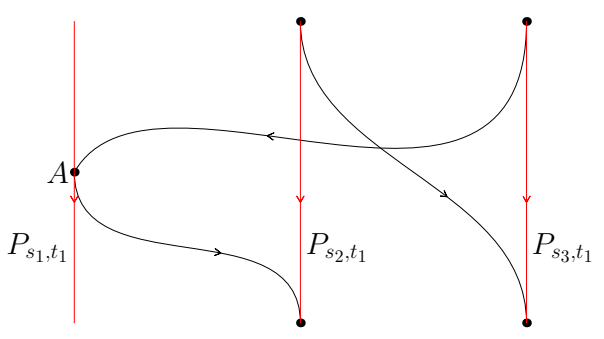

(b)

Figure 4: If we reverse the directions of all the $\mathbf{P}_{t_{1}}$-paths, then both $(a)$ and $(b)$ will give rise to directed cycles of $\mathcal{N}_{1}$. Note that the one from $(a)$ is regular, whereas the one from $(b)$ is singular since it contains an isolated vertex $A$ on the path $P_{s_{1}, t_{1}}$. And by definition, (a) is a $\mathbf{P}_{t_{1}}$-semi-cycle.

and $\mathbf{P}_{t_{3}}^{(2)}=\left\{P_{s_{1}, t_{3}}, P_{s_{2}, t_{3}}^{(2)}, P_{s_{3}, t_{3}}\right\}$. By definition, $\left\{\left[s_{2}, D, F, I\right],\left[s_{2}, E, G, I\right]\right\}$ is a $\mathbf{P}_{t_{3}}^{(1)}$-semicycle and also a $\mathbf{P}_{t_{3}}^{(2)}$-semi-cycle of $\mathcal{N} ;\left[s_{2}, E, G, I\right]$ is a $\mathbf{P}_{t_{3}}^{1}$-crossing and $\left[s_{2}, D, F, I\right]$ is a $\mathbf{P}_{t_{3}}^{(2)}$-crossing. If we reverse the direction of $\mathbf{P}_{t_{3}}^{(1)}$, then $\left[s_{2}, E, G, I, F, D, s_{2}\right]$ is a cycle in the corresponding residual network, and if we choose to reverse the direction of $\mathbf{P}_{t_{3}}^{(2)}$, then $\left[s_{2}, D, F, I, G, E, s_{2}\right]$ is a cycle in the corresponding residual network.

We are now ready to state the following theorem, which give characterizations of stable networks.

Theorem 3.5. For any extra strongly reachable $k$-pair network $\mathcal{N}$, the following statements are all equivalent.

1) $\mathcal{N}$ is stable.

2) $\mathcal{N}$ has no $\mathbf{P}_{t_{j}}$-semi-cycle, $j=1,2, \ldots, k$.

3) $\mathcal{N}$ has no $\mathbf{P}_{t_{j}}$-crossing, $j=1,2, \ldots, k$.

4) None of $\mathcal{N}_{1}, \mathcal{N}_{2}, \ldots, \mathcal{N}_{k}$ has a regular directed cycle.

Proof. We will only establish the equivalence between 1) and 2), which is the only non-trivial part of the proof.

$2) \rightarrow 1$ ): Suppose $\mathcal{N}$ is unstable. Then, for some $j$, there exist two choices for $\mathbf{P}_{t_{j}}: \mathbf{P}_{t_{j}}^{(1)}=$ $\left\{P_{s_{1}, t_{j}}^{(1)}, P_{s_{2}, t_{j}}^{(1)}, \ldots, P_{s_{k}, t_{j}}^{(1)}\right\}$ and $\mathbf{P}_{t_{j}}^{(2)}=\left\{P_{s_{1}, t_{j}}^{(2)}, P_{s_{2}, t_{j}}^{(2)}, \ldots, P_{s_{k}, t_{j}}^{(2)}\right\}$. Let $C_{1}$ be the subnetwork of $\mathcal{N}$ induced on $\mathbf{P}_{t_{j}}^{(1)} \cup \mathbf{P}_{t_{j}}^{(2)}$ after removing all the vertices whose in-degree and out-degree are both 1. Then, for each arc $a$ of $C_{1}$, there are three cases: (1) $a$ only belongs to a path of $\mathbf{P}_{t_{j}}^{(1)}$; (2) a only belongs to a path of $\mathbf{P}_{t_{j}}^{(2)} ;(3)$ a belongs to both a path of $\mathbf{P}_{t_{j}}^{(1)}$ and a path of $\mathbf{P}_{t_{j}}^{(1)}$. Let $C$ be the digraph induced on the arcs of Cases (1) and (2) after reversing the directions of the arcs of Case (1). It is easy to see that for each vertex $v$ of $C$, the in-degree of $v$ equals the out-degree of $v$. Thus, $C$ is an Eulerian directed graph and hence composed of arc-disjoint directed cycles, which corresponds to a $\mathbf{P}_{t_{j}}^{(1)}$-semi-cycle by definition.

$1) \rightarrow 2$ ): Suppose that there exists a $\mathbf{P}_{t_{j}}$-semi-cycle $C$, and let $C^{\prime}$ be the corresponding $\mathbf{P}_{t_{j}}$-crossing. Then it is easy to see that $\left(\mathbf{P}_{t_{j}} \backslash C\right) \cup C^{\prime}$ is an alternative choice of $\mathbf{P}_{t_{j}}$, which means that $\mathcal{N}$ is not stable. 
We would like to add that one can efficiently check that if a given $k$-pair network $\mathcal{N}$ is extra strongly reachable by applying the Ford-Fulkerson algorithm to a set of $k$ directed graphs $D_{i}, 1 \leq i \leq k$, constructed below:

- Add a vertex $s$ as the source node and add an arc $\left[s, s_{j}\right]$ for each $s_{j} \in S, 1 \leq j \leq k$.

- Split each vertex $v \in V \backslash\left\{s, t_{i}\right\}$ into two vertices $v_{\text {in }}$ and $v_{\text {out }}$ and add an arc $\left[v_{\text {in }}, v_{\text {out }}\right]$. Accordingly, replace arcs $[s, u],[u, v]$ and $\left[v, t_{i}\right]$ by $\left[s, u_{i n}\right],\left[u_{\text {out }}, v_{\text {in }}\right]$ and $\left[v_{\text {out }}, t_{i}\right]$, respectively.

It is easy to see that the maximal flow from $s$ to $t_{i}$ is the number of vertex-disjoint $\mathbf{P}_{t_{i}}$-paths, and moreover, if it equals $k$ for all $D_{i}$, then $\mathcal{N}$ is extra strongly reachable. Furthermore, it is widely known [5] that the depth-first search (DFS) algorithm can be used to detect directed cycles in a directed graph, which can be slightly modified 2 to detect regular cycles in a residual network $\mathcal{N}_{j}$. To sum up, the equivalence between 1) and 4) of Theorem 3.5, together with the Ford-Fulkerson algorithm and the DFS algorithm, can be used to efficiently check the stability of a $k$-pair network.

\section{Stable 3-pair Networks}

In this section, unless specified otherwise, we assume that $\mathcal{N}$ is a stable 3 -pair network. For the sake of convenience, a $\mathbf{P}_{t_{1}}$-path, $\mathbf{P}_{t_{2}}$-path or $\mathbf{P}_{t_{3}}$-path may be referred to as a red path, green path or blue path, respectively. For each feasible $i$, we may use shorthand notations $r_{i}, g_{i}$ and $b_{i}$ for paths $P_{s_{i}, t_{1}}, P_{s_{i}, t_{2}}$ and $P_{s_{i}, t_{3}}$, respectively. Similarly, we call a $\mathbf{P}_{t_{1}}$-crossing, $\mathbf{P}_{t_{2}}$-crossing and $\mathbf{P}_{t_{3}}$-crossing as a $r$-crossing, $g$-crossing and $b$-crossing, respectively.

Definition 4.1. [Longest Common Segment (l.c.s.)] For any directed paths $p_{1}, p_{2}, \ldots, p_{r}$ in $\mathcal{N}$, a longest common segment of $\left\{p_{1}, p_{2}, \ldots, p_{r}\right\}$, henceforth abbreviated as a $\left\{p_{1}, p_{2}, \ldots, p_{r}\right\}$ l.c.s., is a segment common to all $p_{i}$ and any segment properly containing it is not common to all $p_{i}$.

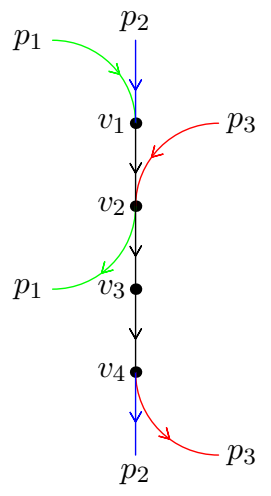

Figure 5: Longest common segments by paths $p_{1}, p_{2}$ and $p_{3}$

\footnotetext{
${ }^{2}$ To see this, whenever the DFS visits a vertex on a $\mathbf{P}_{t_{j}}$-path from a vertex outside of the $\mathbf{P}_{t_{j}}$-path, it goes along the $\mathbf{P}_{t_{j}}$-arc for the next step's visit.
} 
For example, In Fig. 5, there are three paths $p_{1}, p_{2}, p_{3}$ represented using distinct colors. It is easy to see that $\left[v_{1}, v_{2}\right]$ is a $\left\{p_{1}, p_{2}\right\}$-l.c.s.; $\left[v_{2}, v_{3}, v_{4}\right]$ is a $\left\{p_{2}, p_{3}\right\}$-1.c.s.; vertex $v_{2}$ is a $\left\{p_{1}, p_{2}, p_{3}\right\}$-l.c.s. and also a $\left\{p_{1}, p_{3}\right\}$-l.c.s. On the other hand, $\left[v_{2}, v_{3}\right]$ is not a $\left\{p_{2}, p_{3}\right\}$-l.c.s. since $\left[v_{2}, v_{3}, v_{4}\right]$, which properly contains $\left[v_{2}, v_{3}\right]$, is common to both $p_{2}$ and $p_{3}$.

\section{1 $\mathcal{N}_{t_{i}, t_{j}}$}

For $i \neq j$, we will use $\mathcal{N}_{t_{i}, t_{j}}$ to denote the subnetwork of $\mathcal{N}$ induced on all $\mathbf{P}_{t_{i}}$-paths and $\mathbf{P}_{t_{j}}$-paths. In this section, we will characterize the topology of $\mathcal{N}_{t_{i}, t_{j}}$, and without loss of generality, we will only consider $\mathcal{N}_{t_{1}, t_{2}}$. For any path $p \in \mathbf{P}_{t_{1}} \cup \mathbf{P}_{t_{2}}=\left\{r_{1}, r_{2}, r_{3}, g_{1}, g_{2}, g_{3}\right\}$, let $\ell(p)$ denote the number of $\left\{r_{i}, g_{j}\right\}$-l.c.s.'s $(1 \leq i, j \leq 3)$ on $p$ and we order all such l.c.s.'s by $p(1)<p(2)<\cdots<p(\ell(p))$, where by $p(i)<p(i+1)$, we mean head $(p(i))<\operatorname{tail}(p(i+1))$ according to the topological order of the vertices/arcs of a DAG; and we will use $p(i, i+1)$ to denote the path segment of $p$ form head $(p(i))$ to $\operatorname{tail}(p(i+1))$. Note that $r_{j}(1)=g_{j}(1)$ since $r_{j}$ and $g_{j}$ share the same source $s_{j}$ for all feasible $j$. We first give a simple yet very useful lemma.

Lemma 4.2. For $p, q \in\left\{r_{1}, r_{2}, r_{3}, g_{1}, g_{2}, g_{3}\right\}$ and $l=1,2, \ldots, \ell(p)-1$, if $p(l) \subseteq q$, then $p(l+1) \nsubseteq q$.

Proof. Without loss of generality, we suppose $q \in\left\{r_{1}, r_{2}, r_{3}\right\}$. Clearly, if $p(l), p(l+1) \subseteq q$, then $p(l, l+1)$ forms a $r$-crossing, which contradict the assumption that the network is stable.

The following lemma is a key tool in this paper.

Lemma 4.3. There exist $1 \leq i \neq j \leq 3$ such that $\ell\left(r_{i}\right)=\ell\left(g_{j}\right)=1$.

Proof. (1) We first prove that there exists a green path $g_{j}$ such that $\ell\left(g_{j}\right)=1$. To this end, note that if for all $1 \leq i \leq 3, \ell\left(g_{i}\right)=1$, then the desired result is obviously true. Hence, we suppose, without loss of generality, that $\ell\left(g_{1}\right)>1$. Clearly, by Lemma 4.2, $g_{1}(2) \nsubseteq r_{1}$. Thus, we further assume in the following that $g_{1}(2) \subseteq r_{2}$ (See Fig. [6) (a)).

Now, we consider $g_{2}$. If $\ell\left(g_{2}\right)=1$, then we are done. So we suppose in the following that $\ell\left(g_{2}\right)>1$. Then, by Lemma 4.2, we deduce that $g_{2}(2) \nsubseteq r_{2}$. We also have that $g_{2}(2) \nsubseteq r_{1}$ since otherwise $g_{1}(1,2)$ and $g_{2}(1,2)$ form a $r$-crossing. So, we have $g_{2}(2) \subseteq r_{3}$ (See Fig. 6(b)).

Now, consider $g_{3}$ and suppose, by way of contradiction, that $\ell\left(g_{3}\right)>1$. Then, we have (1) $g_{3}(2) \nsubseteq r_{3}$ (by Lemma 4.2); (2) $g_{3}(2) \nsubseteq r_{2}$ since otherwise $g_{2}(1,2)$ and $g_{3}(1,2)$ form a $r$-crossing; and $(3) g_{3}(2) \nsubseteq r_{1}$ since otherwise $g_{1}(1,2), g_{2}(1,2)$ and $g_{3}(1,2)$ form a $r$-crossing. Hence, we obtain a contradiction to the existence of $g_{3}(2)$ and thus deduce that $\ell\left(g_{3}\right)=1$, completing the proof of (1).

(2) By considering the red paths in the parallel manner, we can find a red path, say, $r_{i}$, such that $\ell\left(r_{i}\right)=1$.

(3) We now prove $i \neq j$ by contradiction. Without loss of generality, we suppose $i=j=$ 1, i.e., $\ell\left(r_{1}\right)=\ell\left(g_{1}\right)=1$. Note that if $\ell\left(g_{2}\right)=1$, then we are done. Hence, we suppose in the following that $\ell\left(g_{2}\right)>1$. Clearly, $g_{2}(2) \nsubseteq r_{2}$ by Lemma 4.2 and $g_{2}(2) \nsubseteq r_{1}$ since $\ell\left(r_{1}\right)=1$. Hence, $g_{2}(2) \subseteq r_{3}$.

Now, consider $g_{3}$. If $\ell\left(g_{3}\right)=1$, then we are done since $\ell\left(r_{1}\right)=\ell\left(g_{3}\right)=1$. So, we suppose $\ell\left(g_{3}\right)>1$. Clearly, $g_{3}(2) \nsubseteq r_{3}$ by Lemma 4.2 , and $g_{3}(2) \nsubseteq r_{2}$ since otherwise $g_{2}(1,2)$ and 
$g_{3}(1,2)$ form a $r$-crossing. Note that $\ell\left(r_{1}\right)=1$, we have $g_{3}(2) \nsubseteq r_{1}$, which implies that $\ell\left(g_{3}\right)=1$, completing the proof of the lemma.

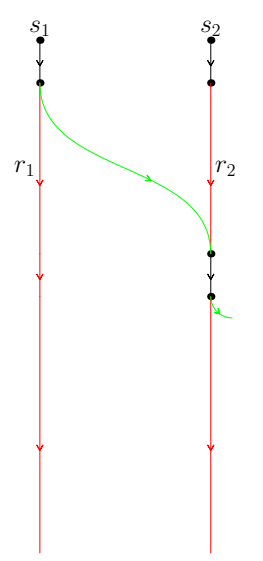

$(a)$

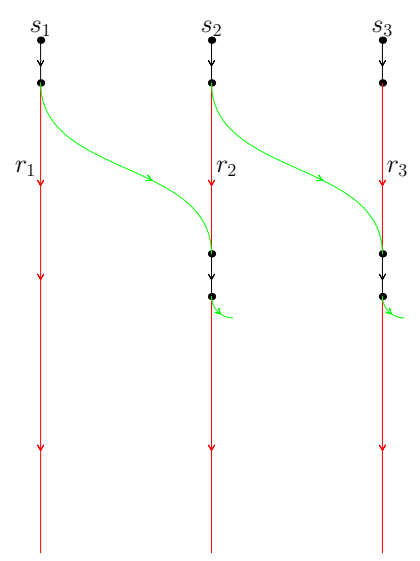

(b)

Figure 6: Proof of Lemma 4.3.

A careful examination of the above proof, in particular, Step (3) thereof, reveals that it actually yields a stronger result:

Corollary 4.4. If there exists a feasible $i$ such that $\ell\left(r_{i}\right)=1$ (resp. $\ell\left(g_{i}\right)=1$ ), then there exists a feasible $j$ such that $j \neq i$ and $\ell\left(g_{j}\right)=1$ (resp. $\left.\ell\left(r_{j}\right)=1\right)$.

Definition 4.5. [Non-degenerated $\mathcal{N}_{t_{1}, t_{2}}$ ] We say $\mathcal{N}_{t_{1}, t_{2}}$ is non-degenerated if there uniquely exist distinct $i, j$ such that $\ell\left(r_{i}\right)=\ell\left(g_{j}\right)=1$, otherwise degenerated.

The following corollary lists all possible topologies of a degenerated $\mathcal{N}_{t_{1}, t_{2}}$.

Theorem 4.6. A degenerated $\mathcal{N}_{t_{1}, t_{2}}$ is equivalent to $(a),(b)$, or $(c)$ of Fig. 7 in the sense that the two are isomorphic if each l.c.s. is treated as a single vertex.

Proof. We will have to deal with the following two cases:

1) there exists $i$ such that $\ell\left(r_{i}\right)=\ell\left(g_{i}\right)=1$. In this case, by Corollary 4.4, we have the following subcases:

1.1) There exists $j \neq i$ such that $\ell\left(g_{j}\right)=1 ; \ell\left(r_{j}\right)=1$. In this case, it is easy to see that there exists $l$ distinct from both $i$ and $j$ such that $\ell\left(r_{l}\right)=\ell\left(g_{l}\right)=1$ as shown in $(a)$ of Fig. 7 .

1.2) There exist $j \neq i$ and $l \neq i$ such that $\ell\left(r_{j}\right)=1 ; \ell\left(g_{l}\right)=1$. In this case, if $\ell\left(g_{j}\right)=1$, we have Case 1.1); otherwise, we have $g_{j}(2)=r_{l}(2)$ as shown in $(b)$ of Fig. 7 .

2) there exist distinct $i, j, l$ such that $\ell\left(r_{i}\right)=\ell\left(r_{j}\right)=\ell\left(g_{l}\right)=1$. In this case, we have either $r_{l}(2)=g_{j}(2) ; r_{l}(3)=g_{i}(2)$ as shown in $(c)$ of Fig. 7 or $r_{l}(2)=g_{i}(2) ; r_{l}(3)=g_{j}(2)$ resulting a network equivalent to $(c)$.

The proof is complete by combining all the discussions above. 


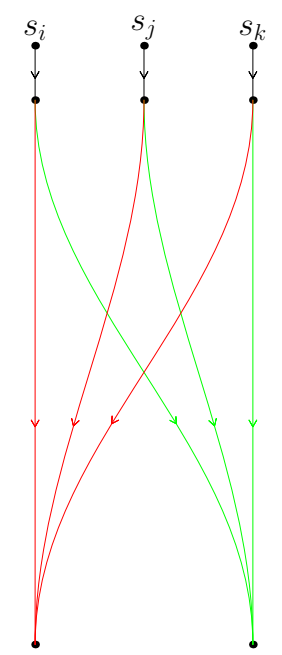

(a)

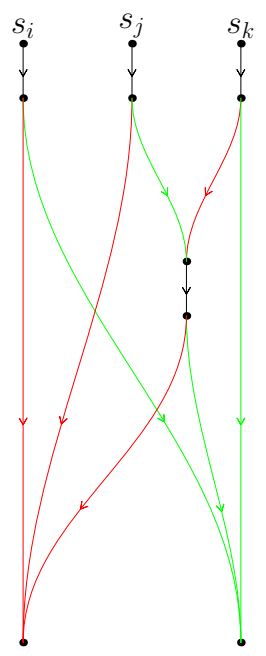

(b)

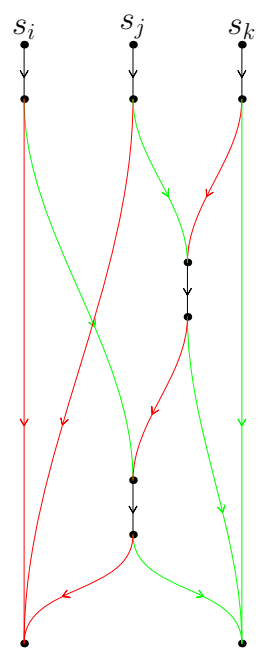

(c)

Figure 7: Possible cases of a degenerated $\mathcal{N}_{t_{1}, t_{2}}$.

Theorem 4.7. A non-degenerated $\mathcal{N}_{t_{1}, t_{2}}$ is equivalent to one of the five networks as shown in Fig. 8 in the sense that the two are isomorphic if each l.c.s. is treated as a single vertex.

Proof. For a non-degenerated $\mathcal{N}_{t_{1}, t_{2}}$, we suppose that $\ell\left(r_{i}\right)=\ell\left(g_{l}\right)=1$ and consider all the possible l.c.s. of $\left\{r_{j}, r_{l}\right\}$ and $\left\{g_{i}, g_{j}\right\}$. Recalling that $r_{l}(1)=g_{l}(1)$, for all feasible $l$, we start our argument by considering $g_{i}(2)$ and $g_{j}(2)$. By Lemma 4.2, we have the following two cases:

1) $g_{i}(2) \subseteq r_{j}$ and $g_{j}(2) \subseteq r_{l}$. In this case, by Lemma 4.2, we infer that $r_{j}(2) \nsubseteq g_{j}$ and hence $r_{j}(2) \subseteq g_{i}$, which implies that $g_{i}(2)=r_{j}(2)$ (due to the acyclicity of $\mathcal{N}$ ). We then further consider the following two subcases:

1.1) $\ell\left(g_{i}\right)=2$.

1.2) $\ell\left(g_{i}\right)>2$.

In Case 1.1), it is easy to see that $g_{j}(2)=r_{l}(2)$, which leads to the following two subcases:

1.1.1) $\ell\left(g_{j}\right)=2$. In this case, $\mathcal{N}_{t_{1}, t_{2}}$ is equivalent to (1.1.1) of Fig. 8.

1.1.2) $\ell\left(g_{j}\right) \geq 3$. In this case, $g_{j}(3) \subseteq r_{j}$. By Lemma 4.2 and the acylicity of the network, we have $g_{j}(3)=r_{j}(3)$. We declare that $\ell\left(g_{j}\right)=3$ since if otherwise $g_{j}(4) \subseteq r_{l}$, again by Lemma 4.2 and the acyclicity of the network, we have $g_{j}(4)=$ $r_{l}(3)$ and hence $r_{l}(2), r_{l}(3) \subseteq g_{j}$, which contradicts Lemma 4.2. Hence, in this case, $\mathcal{N}_{t_{1}, t_{2}}$ is equivalent to (1.1.2) of Fig. 8 .

In Case 1.2), since $g_{i}(2) \subseteq r_{j}$, we have $g_{i}(3) \subseteq r_{l}$. Since $g_{j}(2), g_{i}(3) \subseteq r_{l}$, we have to deal with the following two subcases:

1.2.1) $g_{j}(2)=r_{l}(2)$ and $g_{i}(3)=r_{l}(3)$. In this case, if $\ell\left(g_{j}\right) \geq 3$, then by Lemma 4.2 , $g_{j}(3) \subseteq r_{j}$, which however would imply $g_{j}(2,3)$ and $g_{i}(2,3)$ form a $r$-crossing. 
Hence, we have $\ell\left(g_{j}\right)=2$. Now, if $\ell\left(g_{i}\right) \geq 4$, then by Lemma 4.2, $g_{i}(4) \subseteq r_{j}$, which further implies $g_{i}(4)=r_{j}(3)$. Hence, $r_{j}(2), r_{j}(3) \subseteq g_{i}$, which contradicts Lemma 4.2, Hence $\ell\left(g_{j}\right)=2, \ell\left(g_{i}\right)=3$, and $\mathcal{N}_{t_{1}, t_{2}}$ is equivalent to (1.2.1) of Fig. 8 ,

1.2.2) $g_{i}(3)=r_{l}(2)$ and $g_{j}(2)=r_{l}(3)$. In this case, if $\ell\left(g_{i}\right) \geq 4$, then $g_{i}(4) \subseteq r_{j}$ and hence $g_{i}(3,4)$ and $g_{j}(1,2)$ form a $r$-crossing, which contradicts the stability of the network. Thus, $\ell\left(g_{i}\right)=3$ and we have to consider the following two subcases:

1.2.2.1) $\ell\left(g_{j}\right)=2$. In this case, we conclude that $\mathcal{N}_{t_{1}, t_{2}}$ is equivalent to (1.2.2.1) of Fig. 8.

1.2.2.2) $\ell\left(g_{j}\right) \geq 3$. In this case, by Lemma 4.2, we have $g_{j}(3) \subseteq r_{j}$, which further implies $g_{j}(3)=r_{j}(3)$. Now, if $\ell\left(g_{j}\right) \geq 4$, then by Lemma 4.2, $g_{j}(4) \subseteq r_{l}$, which further implies $g_{j}(4)=r_{l}(4)$ and hence $r_{l}(3), r_{l}(4) \subseteq g_{j}$, which contradicts Lemma 4.2. Hence $\ell\left(g_{j}\right)=3$ and we conclude that $\mathcal{N}_{t_{1}, t_{2}}$ is equivalent to (1.2.2.2) of Fig. 8 .

2) $g_{i}(2) \subseteq r_{l}$ and $g_{j}(2) \subseteq r_{l}$. In this case, without loss of generality, we assume $g_{j}(2)=r_{l}(2)$ and $g_{i}(2)=r_{l}(3)$ (since otherwise, we can relabel $s_{i}, s_{j}$ as $s_{j}, s_{i}$, respectively). By Lemma 4.2, we have $g_{i}(3) \subseteq r_{j}$ and $g_{j}(3) \subseteq r_{j}$. Then, there are two cases:

2.1) $g_{j}(3)=r_{j}(2)$;

2.2) $g_{i}(3)=r_{j}(2)$.

It is easy to see that 2.1) is impossible since otherwise $r_{j}(1), r_{j}(2) \subseteq g_{j}$, which contradicts Lemma 4.2. Hence, we have $r_{j}(2) \subseteq g_{i}$ and $r_{l}(2) \subseteq g_{j}$. By switching the colors of the paths and relabeling sources $s_{i}, s_{l}$ as $s_{l}, s_{i}$, respectively, we will reach Case 1), which has been dealt with before.

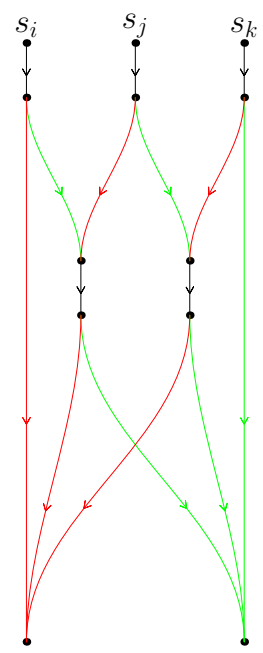

$(1.1 .1)$

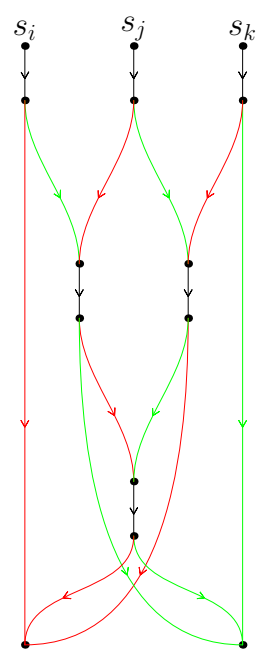

(1.1.2)

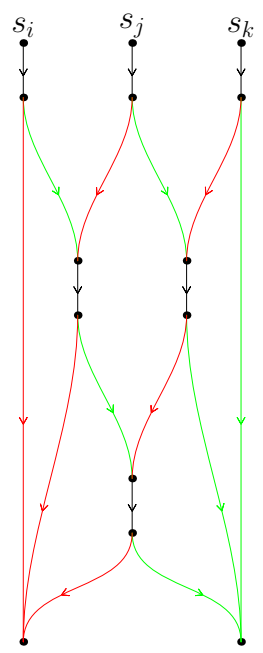

(1.2.1)

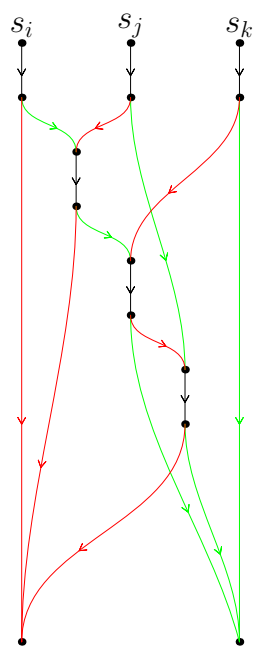

(1.2.2.1)

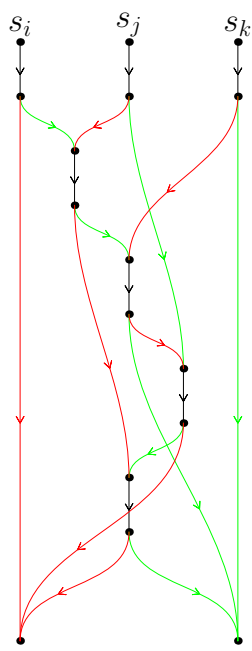

(1.2.2.2)

Figure 8: Possible cases of a non-degenerated $\mathcal{N}_{t_{1}, t_{2}}$. 
The following corollary follows from an inspection of all the possible cases of a nondegenerated $\mathcal{N}_{t_{1}, t_{2}}$ as stated in Theorem 4.7.

Corollary 4.8. Suppose that $\mathcal{N}_{t_{1}, t_{2}}$ is non-degenerated with $\ell\left(r_{i}\right)=\ell\left(g_{l}\right)=1$. Then, (1) $g_{i}(2) \neq r_{l}(2)$; (2) there exist a unique $\left\{g_{i}, r_{j}\right\}$-l.c.s. and a unique $\left\{g_{j}, r_{l}\right\}$-l.c.s., where $j$ is distinct from both $i$ and $l$; and (3) one of the following 3 statements holds:

a) $g_{i}(2)=r_{j}(2)$ and $g_{j}(2)=r_{l}(2)$;

b) $g_{i}(2)=r_{j}(2)$ and $g_{j}(2)=r_{l}(3)$;

c) $g_{i}(3)=r_{j}(2)$ and $g_{j}(2)=r_{l}(2)$.

We say $\mathcal{N}_{t_{1}, t_{2}}$ is of type 1 if $a$ ) of Corollary 4.8 holds, and of type 2 otherwise. It is easy to check that in Fig. 8, (1.1.1), (1.1.2), (1.2.1) are of type 1 and $(1,2,2,1),(1,2,2,2)$ are of type 2 since they satisfy $b)$. Note that in Fig. 8, if we switch colors of paths and source labels $i$ and $l$, then (1.1.1), (1.1.2), (1.2.1) still satisfy $a)$ but $(1,2,2,1),(1,2,2,2)$ satisfy $c)$ of Corollary 4.8 instead.

\subsection{A Forbidden Structure}

For any $\mathbf{P}$-path $p$ in $\mathcal{N}_{t_{i}, t_{j}}$, let $\ell^{i, j}(p)$ be the number of $\left\{P_{s_{l}, t_{i}}, P_{s_{l^{\prime}}, t_{j}}\right\}$-l.c.s's $\left(1 \leq l, l^{\prime} \leq 3\right)$ on $p$ and we order such l.c.s's as $p^{i, j}(1)<p^{i, j}(2)<\cdots<p^{i, j}\left(\ell^{i, j}(p)\right)$. Here we remark that the notation $\ell^{i, j}(p), p^{i, j}(\cdot)$ subsume $\ell(p), p(\cdot)$ as the latter two are simply $\ell^{1,2}(p), p^{1,2}(\cdot)$, respectively. By Lemma 4.3, the following sets are non-empty:

$$
m_{i, j}^{i}:=\left\{l: \ell^{i, j}\left(P_{s_{l}, t_{i}}\right)=1\right\}, \quad m_{i, j}^{j}:=\left\{l: \ell^{i, j}\left(P_{s_{l}, t_{j}}\right)=1\right\} .
$$

Here, let us add that the two subscripts of $m_{i, j}^{i}$ are interchangeable, more precisely, $m_{i, j}^{i}=$ $m_{j, i}^{i}$. In the case $m_{i, j}^{i}$ contains only one element, e.g., $\mathcal{N}_{t_{i}, t_{j}}$ is degenerated, we may write $m_{i, j}^{i}=l$ instead of $m_{i, j}^{i}=\{l\}$ for simplicity. For example, for the network depicted in Fig. 1(a), each $\mathcal{N}_{t_{i}, t_{j}}$ is non-degenerated and $m_{1,2}^{1}=1, m_{1,2}^{2}=3, m_{1,3}^{1}=1, m_{1,3}^{3}=3$, $m_{2,3}^{2}=1$ and $m_{2,3}^{3}=3$.

Theorem 4.9. There exists no stable network such that $(1) m_{i, j}^{i}=l, m_{i, j}^{j}=i ; m_{i, l}^{i}=l$, $m_{i, l}^{l}=j ; m_{j, l}^{j}=i, m_{j, l}^{l}=j ;(2)$ there exists a $\left\{P_{s_{j}, t_{i}}, P_{s_{l}, t_{j}}, P_{s_{i}, t_{l}}\right\}$-l.c.s, where $i, j, l$ are all distinct from one another.

Proof. Suppose, by way of contradiction, that there exists a stable network $\mathcal{N}$ such that (1) and (2) hold. Without loss of generality, we assume $i=1, j=2$ and $l=3$ and therefore $m_{1,2}^{1}=m_{1,3}^{1}=3, m_{2,3}^{2}=m_{1,2}^{2}=1, m_{2,3}^{3}=m_{1,3}^{3}=2$, which implies $\ell^{1,2}\left(r_{3}\right)=\ell^{1,3}\left(r_{3}\right)=1$, $\ell^{1,2}\left(g_{1}\right)=\ell^{2,3}\left(g_{1}\right)=1$ and $\ell^{1,3}\left(b_{2}\right)=\ell^{2,3}\left(b_{2}\right)=1$. We consider the following two cases:

1) all $\mathcal{N}_{t_{1}, t_{2}}, \mathcal{N}_{t_{1}, t_{3}}$ and $\mathcal{N}_{t_{2}, t_{3}}$ are of type 1 ;

2) any of $\mathcal{N}_{t_{1}, t_{2}}, \mathcal{N}_{t_{1}, t_{3}}$ or $\mathcal{N}_{t_{2}, t_{3}}$ is of type 2. 


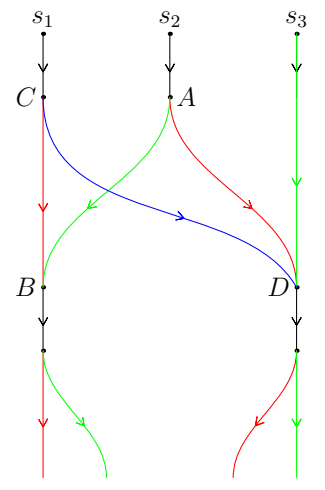

(a)

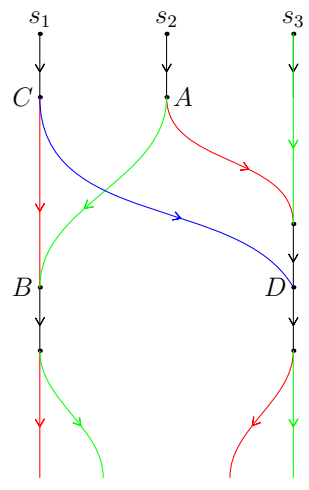

(b)

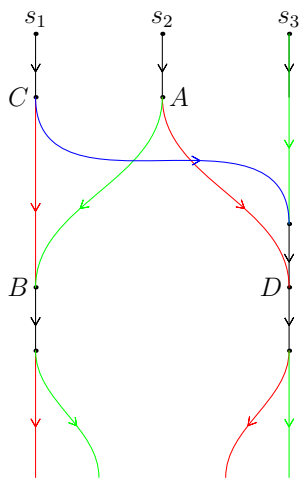

$(c)$

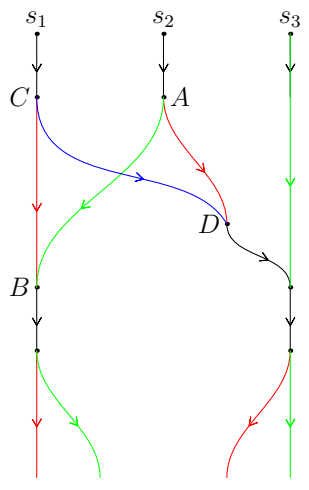

$(d)$

Figure 9: Proof of Case 1) of Theorem 4.9, where we do not show path $b_{3}$. Note that $r_{2}^{1,2}(2)=g_{3}^{1,2}(2), r_{2}^{1,3}(2)=b_{1}^{1,3}(2), g_{3}^{2,3}(2)=b_{1}^{2,3}(2)$ and there exists a unique $\left\{r_{2}, g_{3}, b_{1}\right\}$-l.c.s.

We first prove the theorem for Case 1). Consider $\mathcal{N}_{t_{1}, t_{2}}$. Since it is of type 1 , by Lemma 4.8, we have that $r_{1}^{1,2}(2)=g_{2}^{1,2}(2), r_{2}^{1,2}(2)=g_{3}^{1,2}(2)$. Note that although there are several types of $\left\{r_{2}, g_{3}, b_{1}\right\}$-l.c.s., as shown in $(a)-(d)$ of Fig. 9, our argument in the following however does not depend on the specific type. In the following, we prove $g_{2}^{1,2}(1,2)$ and $b_{1}^{1,3}(1,2)$ (shown as $[A, B]$ and $[C, D]$, respectively in Fig. 9) form a $r$-crossing, which will contradict Theorem 3.5 and yield the theorem for this case. Towards this goal, we only need to prove the following two statements:

(a) $C<B$ and $A<D$;

(b) $g_{2}^{1,2}(1,2) \cap b_{1}^{1,3}(1,2)=\emptyset$.

For $(a)$, it is easy to see that either $B \leq C$ or $D \leq A$ will imply that $b_{1}^{2,3}(2) \subseteq g_{2}$, which contradicts the fact that $b_{1}^{2,3}(2)=g_{3}^{2,3}(2)$ since $\mathcal{N}_{t_{2}, t_{3}}$ is of type 1 . Hence $(a)$ holds. For $(b)$, it is easy to see that $g_{2}^{1,2}(1,2) \cap b_{1}^{1,3}(1,2) \neq \emptyset$ also contradicts the fact that $b_{1}^{2,3}(2)=g_{3}^{2,3}(2)$. Hence, (b) holds.

Now, we prove the theorem for Case 2). Without loss of generality, we suppose $\mathcal{N}_{t_{1}, t_{2}}$ is of type 2. Then, according to Corollary 4.8, there are two possible cases. Specifically, in Fig. 10 (resp. Fig. 11), (a) satisfies: $r_{1}^{1,2}(2)=g_{2}^{1,2}(2)$ and $r_{2}^{1,2}(2)=g_{3}^{1,2}(3)$; and (b) satisfies: $r_{1}^{1,2}(3)=g_{2}^{1,2}(2)$ and $r_{2}^{1,2}(2)=g_{3}^{1,2}(2)$.

We consider the following two cases:

2.1) $b_{1}^{2,3}(2) \subseteq g_{3}$;

2.2) $b_{1}^{2,3}(2) \subseteq g_{2}$.

For Case 2.1) (see Fig. 10), the proof is similar to that of Case 1). In this case, we can have $g_{2}^{1,2}(1,2)$ and $b_{1}^{1,3}(1,2)$ (shown as $[A, B]$ and $[C, D]$, respectively in Fig. 10) form a $r$-crossing, which contradicts the stability of the network.

For Case 2.2), since $b_{1}^{2,3}(2) \neq g_{2}^{2,3}(2)$ and $b_{1}^{2,3}(2) \nsubseteq g_{3}$, we have, by Lemma $4.8, b_{1}^{2,3}(2)=$ $g_{2}^{2,3}(3)$ and $b_{3}^{2,3}(2)=g_{2}^{2,3}(2)$, as shown in Fig. 11]. Let $A:=\operatorname{head}\left(r_{2}^{1,2}(1)\right), B:=\operatorname{tail}\left(r_{2}^{1,2}(2)\right)$, $C:=\operatorname{head}\left(b_{3}^{2,3}(1)\right), D:=\operatorname{head}\left(b_{3}^{2,3}(2)\right)$. Noticing that either $B \leq C$ or $D \leq A$ or $r_{2}^{1,2}(1,2) \cap$ 


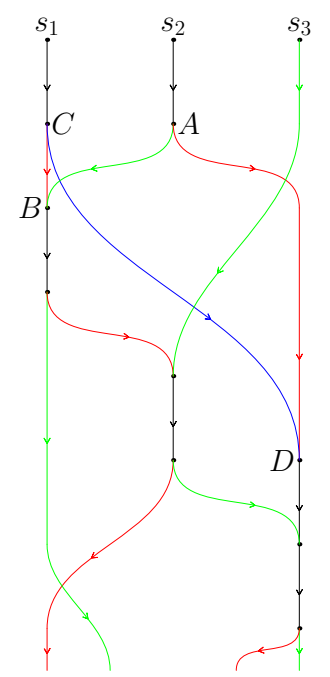

(a)

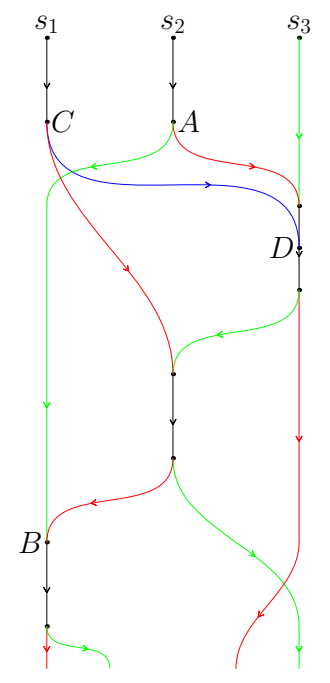

(b)

Figure 10: Proof of Case 2.1) of Theorem 4.9, where we do not show path $b_{3}$.

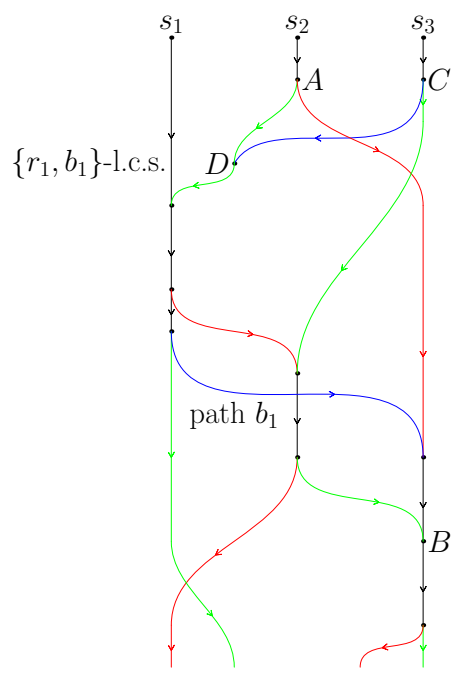

(a)

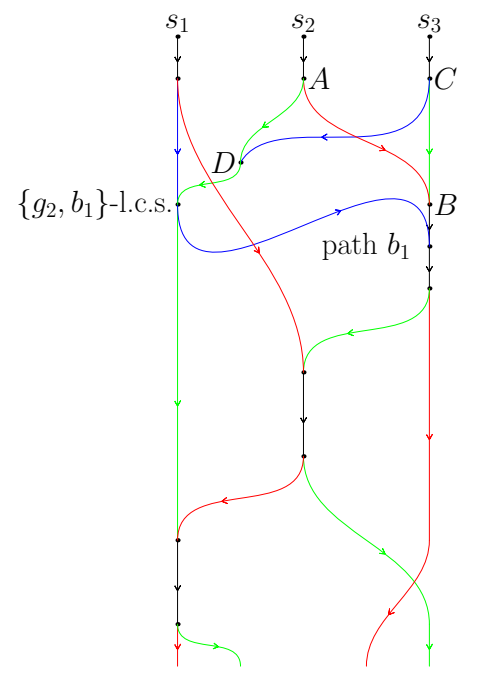

(b)

Figure 11: Proof of Case 2.2) of Theorem 4.9. 
$b_{3}^{2,3}(1,2) \neq \emptyset$ will imply that $r_{2}^{1,3}(2)=b_{3}^{1,3}(2)$, which however, is impossible according to $(1)$ of Corollary 4.8. Hence, we have

(a) $C<B$ and $A<D$;

(b) $r_{2}^{1,2}(1,2) \cap b_{3}^{2,3}(1,2)=\emptyset$.

By definition, $r_{2}^{1,2}(1,2)$ and $b_{3}^{2,3}(1,2)$ form a $g$-crossing, a contradiction that leads to the theorem for this case.

The proof is then complete by combining all the discussions above.

\section{$5 \quad$ Main Result}

In this section, we state and prove our main result. Throughout this section, we again assume that $\mathcal{N}$ is a stable 3 -pair network.

The following seemingly trivial lemma is a key tool for us to determine $\mathcal{S}_{\mathcal{N}}$ throughout our treatment.

Lemma 5.1. If there are no $\left\{P_{s_{i_{1}}, t_{j_{1}}}, P_{s_{i_{2}}, t_{j_{2}}}\right\}$-l.c.s. within $\mathcal{N}$, then $\left\{\left(i_{1}, j_{1}\right),\left(i_{2}, j_{2}\right)\right\} \nsubseteq s$ for any $s \in \mathcal{S}_{\mathcal{N}}$.

The following lemma is useful.

Lemma 5.2. If there exists $h$ such that $h \in m_{i, j}^{l_{1}} \cap m_{i, l}^{l_{2}} \cap m_{j, l}^{l_{3}}$ for some feasible $l_{1}, l_{2}, l_{3}$ and distinct $i, j, l$, then either $\{(h, i),(h, j)\} \notin \mathcal{S}_{\mathcal{N}}$ or $\{(h, i),(h, l)\} \notin \mathcal{S}_{\mathcal{N}}$.

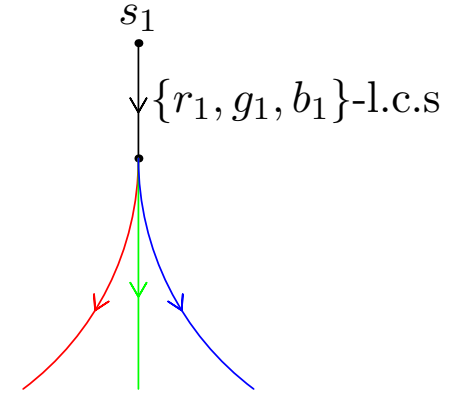

(a)

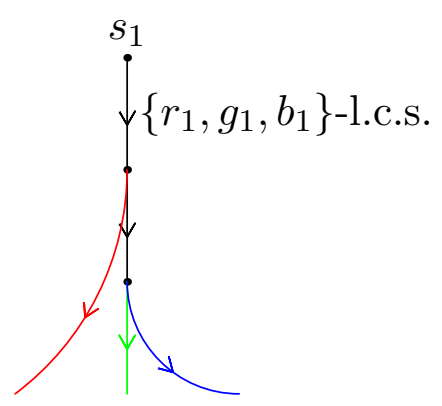

(b)

Figure 12: Proof of Lemma 5.2. In $(a)$, the unique $\left\{r_{1}, g_{1}, b_{1}\right\}$-l.c.s. is also the unique $\left\{r_{1}, g_{1}\right\}$-l.c.s., $\left\{g_{1}, b_{1}\right\}$-l.c.s. and $\left\{r_{1}, b_{1}\right\}$-l.c.s.. In $(b)$, the unique $\left\{r_{1}, g_{1}, b_{1}\right\}$-l.c.s. is also the unique $\left\{r_{1}, g_{1}\right\}$-l.c.s. and $\left\{r_{1}, b_{1}\right\}$-l.c.s..

Proof. Without loss of generality, we assume $h=1$. Then, there exist a unique $\left\{r_{1}, g_{1}\right\}$ l.c.s., a unique $\left\{r_{1}, b_{1}\right\}$-l.c.s. and a unique $\left\{g_{1}, b_{1}\right\}$-l.c.s. with a same tail $s_{1}$. If all of them have a same head, as shown in (a) of Fig. [12, then $\{(1,1),(1,2),(1,3)\} \in \mathcal{S}_{\mathcal{N}}$ and none of $\{(1,1),(1,2)\},\{(1,1),(1,3)\},\{(1,2),(1,3)\}$ belongs to $\mathcal{S}_{\mathcal{N}}$; otherwise two of them share a 
same head, then $\{(1,1),(1,2),(1,3)\} \in \mathcal{S}_{\mathcal{N}}$ and at most one of $\{(1,1),(1,2)\},\{(1,1),(1,3)\}$, $\{(1,2),(1,3)\}$ belongs to $\mathcal{S}_{\mathcal{N}}$ (for example, $(b)$ shows the case $\{(1,2),(1,3)\} \in \mathcal{S}_{\mathcal{N}}$ ). Hence, the result holds for both cases, which completes the proof.

We also need the following lemma.

Lemma 5.3. Let

$$
\mathcal{C}=\left(\left(\begin{array}{ccc}
\frac{1}{2} & \frac{1}{4} & \frac{1}{4} \\
\frac{1}{4} & \frac{-1}{4} & 0 \\
\frac{1}{4} & 0 & \frac{-1}{4}
\end{array}\right),\left(\begin{array}{ccc}
\frac{-1}{4} & \frac{1}{4} & 0 \\
\frac{1}{4} & \frac{1}{2} & \frac{1}{4} \\
0 & \frac{1}{4} & \frac{-1}{4}
\end{array}\right),\left(\begin{array}{ccc}
\frac{-1}{4} & 0 & \frac{1}{4} \\
0 & \frac{-1}{4} & \frac{1}{4} \\
\frac{1}{4} & \frac{1}{4} & \frac{1}{2}
\end{array}\right)\right)
$$

Then for any $s \in \mathcal{S}_{k}, g_{s}(\mathcal{C})>1$ if and only if $\alpha(s)=3$ and $\gamma(s)=0$.

Proof. The result can be obtained by considering the following cases:

1) $\gamma(s)=0$. In this case, it is easy to see that

$$
g_{s}(\mathcal{C})=\frac{1}{4} \sum_{i=1}^{3} m_{\text {Ind }_{s}}(i)=2 \alpha(s)= \begin{cases}\frac{1}{2}, & \alpha(s)=1 \\ 1, & \alpha(s)=2 \\ \frac{3}{2}, & \alpha(s)=3\end{cases}
$$

2) $\gamma(s)=1$. In this case, it is easy to check that

$$
g_{s}(\mathcal{C})= \begin{cases}\frac{1}{2}+\frac{1}{4}+\frac{1}{4}=1, & \alpha(s)=1 \\ \frac{3}{4}+\frac{1}{4}=1, & s=\{(i, i),(i, j)\}, \text { where } i \neq j \\ \frac{1}{2}, & s=\{(i, i),(k, j)\}, \text { where } i, j, k \text { are distinct; } \\ 1, & \alpha(s)=3 .\end{cases}
$$

3) $\gamma(s)=2$. In this case, it is easy to check that $g_{s}(\mathcal{C})=1$.

4) $\gamma(s)=3$. In this case, obviously, $g_{s}(\mathcal{C})=0$.

We are now ready for our main result.

Theorem 5.4. Each stable 3-pair network has a linear routing solution.

Proof. For the stable 3-pair network $\mathcal{N}$, we consider the following two cases:

1) there exist distinct $i, j, l \in\{1,2,3\}, m_{i, j}^{i} \cap\{i, j\} \neq \emptyset$ and $m_{i, l}^{i} \cap\{i, l\} \neq \emptyset$;

2) for any distinct $i, j, l \in\{1,2,3\}$, either $m_{i, j}^{i}=l$ or $m_{i, l}^{i}=j$.

For Case 1), we have the following subcases:

1.1) $i \in m_{i, j}^{i} \cap m_{i, l}^{i}$;

1.2) $j \in m_{i, j}^{i}$ and $l \in m_{i, l}^{i}$; 
1.3) $i \in m_{i, j}^{i}$ and $l \in m_{i, l}^{i}$.

In the following, without loss of generality, we assume $i=1, j=2$ and $l=3$.

For Case 1.1), if $1 \in m_{1,2}^{1} \cap m_{1,3}^{1}$, then $r_{1}$ is disjoint from $\mathcal{N}^{\prime}:=\left\{g_{2}, g_{3}, b_{2}, b_{3}\right\}$, which is a stable (hence extra strongly reachable) 2-pair network. By [3], $\mathcal{N}^{\prime}$ always has a linear routing solution

$$
\left(\left(\begin{array}{cc}
\frac{3}{4} & \frac{1}{4} \\
\frac{1}{4} & \frac{-1}{4}
\end{array}\right),\left(\begin{array}{cc}
\frac{-1}{4} & \frac{1}{4} \\
\frac{1}{4} & \frac{3}{4}
\end{array}\right)\right) .
$$

Hence, $\mathcal{N}$ has the following linear routing solution:

$$
\left(\left(\begin{array}{lll}
1 & 0 & 0 \\
0 & 0 & 0 \\
0 & 0 & 0
\end{array}\right),\left(\begin{array}{ccc}
0 & 0 & 0 \\
0 & \frac{3}{4} & \frac{1}{4} \\
0 & \frac{1}{4} & -\frac{1}{4}
\end{array}\right),\left(\begin{array}{ccc}
0 & 0 & 0 \\
0 & -\frac{1}{4} & \frac{1}{4} \\
0 & \frac{1}{4} & \frac{3}{4}
\end{array}\right)\right) .
$$

For Case 1.2), consider all $s \in \mathcal{S}_{\mathcal{N}} \subseteq \mathcal{S}_{3}$ such that $\alpha(s)=3$. Let $s=\left\{\left(l_{1}, 1\right),\left(l_{2}, 2\right),\left(l_{3}, 3\right)\right\}$. If $l_{1}=1$, then obviously $\gamma(s) \neq 0$; if $l_{1}=2$, then since $2 \in m_{1,2}^{1}$, we have $l_{2}=2$ and hence $\gamma(s) \neq 0$; and if $l_{1}=3$, since $3 \in m_{1,3}^{1}$, we have $l_{3}=3$ and hence $\gamma(s) \neq 0$. Thus, for any $s \in \mathcal{S}_{\mathcal{N}}$ such that $\alpha(s)=3$, we have $\gamma(s) \neq 0$. By Lemma 5.3 ,

$$
\left(\left(\begin{array}{ccc}
\frac{1}{2} & \frac{1}{4} & \frac{1}{4} \\
\frac{1}{4} & \frac{-1}{4} & 0 \\
\frac{1}{4} & 0 & \frac{-1}{4}
\end{array}\right),\left(\begin{array}{ccc}
\frac{-1}{4} & \frac{1}{4} & 0 \\
\frac{1}{4} & \frac{1}{2} & \frac{1}{4} \\
0 & \frac{1}{4} & \frac{-1}{4}
\end{array}\right),\left(\begin{array}{ccc}
\frac{-1}{4} & 0 & \frac{1}{4} \\
0 & \frac{-1}{4} & \frac{1}{4} \\
\frac{1}{4} & \frac{1}{4} & \frac{1}{2}
\end{array}\right)\right) .
$$

is a linear solution of $\mathcal{N}$.

For Case 1.3), since $1 \in m_{1,2}^{1}$ and $3 \in m_{1,3}^{1}$, by Lemma 5.1, we have

$$
\begin{aligned}
\mathcal{S}_{\mathcal{N}} \subseteq \mathcal{S}: & =\{\{(i, j)\}: 1 \leq i, j \leq 3\} \\
& \cup\{\{(i, 1),(j, 2)\}: i=2,3 ; j=1,2,3\} \cup\{\{(1,1),(1,2)\}\} \\
& \cup\{\{(i, 1),(l, 3)\}: i=1,2 ; l=1,2,3\} \cup\{\{(3,1),(3,3)\}\} \\
& \cup\{\{(j, 2),(l, 3)\}: j, l=1,2,3\} \\
& \cup\{\{(1,1),(1,2),(l, 3)\}: l=1,2,3\} \cup\{\{(2,1),(j, 2),(l, 3)\}: j, l=1,2,3\} \\
& \cup\{\{(3,1),(j, 2),(3,3)\}: j=1,2,3\} .
\end{aligned}
$$

Let

$$
\mathcal{C}=\left(\left(\begin{array}{ccc}
\frac{3}{4} & 0 & \frac{1}{4} \\
0 & 0 & 0 \\
\frac{1}{4} & 0 & \frac{-1}{4}
\end{array}\right),\left(\begin{array}{ccc}
0 & 0 & 0 \\
0 & \frac{3}{4} & \frac{1}{4} \\
0 & \frac{1}{4} & \frac{-1}{4}
\end{array}\right),\left(\begin{array}{ccc}
\frac{-1}{4} & 0 & \frac{1}{4} \\
0 & \frac{-1}{4} & \frac{1}{4} \\
\frac{1}{4} & \frac{1}{4} & \frac{1}{2}
\end{array}\right)\right)
$$

Through straightforward computations, one can verify that for any $s \in \mathcal{S}, g_{s}(\mathcal{C}) \leq 1$. Hence, by Theorem 2.5, $\mathcal{C}$ is a linear solution of $\mathcal{N}$, which completes the proof of Case 1 ).

For Case 2), without loss of generality, we assume $m_{i, j}^{i}=l$. Note that by Lemma 4.3, if $m_{i, j}^{i}=l$, then $m_{i, j}^{j} \cap\{i, j\} \neq \emptyset$, which further implies $m_{l, j}^{j}=i$ by the assumption of this case. Hence, by Lemma 4.3, we have $m_{l, j}^{l} \cap\{l, j\} \neq \emptyset$, which implies $m_{l, i}^{l}=j$, again by the assumption of this case, and further implies $m_{l, i}^{i} \cap\{i, l\} \neq \emptyset$ by Lemma 4.3. Finally, we have $l \in m_{i, j}^{i}, j \in m_{i, l}^{l}$ and $i \in m_{j, l}^{j}$. Consider the following subcases:

2.1) $j \in m_{i, j}^{j}, i \in m_{i, l}^{i}$ and $l \in m_{j, l}^{l}$; 
2.2) $i \in m_{i, j}^{j}, i \in m_{i, l}^{i}$ and $l \in m_{j, l}^{l}$;

$\left.2.2^{\prime}\right) j \in m_{i, j}^{j}, i \in m_{i, l}^{i}$ and $j \in m_{j, l}^{l}$;

$\left.2.2^{\prime \prime}\right) j \in m_{i, j}^{j}, l \in m_{i, l}^{i}$ and $l \in m_{j, l}^{l}$;

2.3) $i \in m_{i, j}^{j}, i \in m_{i, l}^{i}$ and $j \in m_{j, l}^{l}$;

2.3') $i \in m_{i, j}^{j}, l \in m_{i, l}^{i}$ and $l \in m_{j, l}^{l}$;

2.3") $j \in m_{i, j}^{j}, l \in m_{i, l}^{i}$ and $j \in m_{j, l}^{l}$;

2.4) $i \in m_{i, j}^{j}, l \in m_{i, l}^{i}$ and $j \in m_{j, l}^{l}$.

It is easy to check that Cases 2.2') and 2.2') can be obtained form Case 2.2) (resp. Cases $2.3^{\prime}$ ) and $2.3^{\prime \prime}$ ) can be obtained form Case 2.3)) by the relabelling: $i \mapsto j, j \mapsto l, l \mapsto i$ and the relabelling: $i \mapsto l, j \mapsto i, l \mapsto j$, respectively. So, in the following, we only need to consider Cases 2.1), 2.2), 2.3), 2.4).

For Case 2.1), without loss of generality, we assume $i=1, j=2$ and $l=3$ and thus $2 \in m_{1,2}^{2}, 1 \in m_{1,3}^{1}$ and $3 \in m_{2,3}^{2}$. Hence, paths $r_{1}, g_{2}$ and $b_{3}$ are pairwise disjoint and the network has a linear routing solution

$$
\left(\left(\begin{array}{lll}
1 & 0 & 0 \\
0 & 0 & 0 \\
0 & 0 & 0
\end{array}\right),\left(\begin{array}{lll}
0 & 0 & 0 \\
0 & 1 & 0 \\
0 & 0 & 0
\end{array}\right),\left(\begin{array}{lll}
0 & 0 & 0 \\
0 & 0 & 0 \\
0 & 0 & 1
\end{array}\right)\right) .
$$

For Case 2.2), without loss of generality, we assume $i=1, j=2$ and $l=3$ and thus $1 \in m_{1,2}^{2} \cap m_{1,3}^{1} \cap m_{2,3}^{2} ; 2 \in m_{1,3}^{3}$ and $3 \in m_{1,2}^{1} \cap m_{2,3}^{3}$. By Lemma [5.1, we have

$$
\begin{aligned}
\mathcal{S}_{\mathcal{N}} \subseteq \mathcal{S}: & =\{\{(i, j)\}: 1 \leq i, j \leq 3\} \\
& \cup\{\{(i, 1),(j, 2)\}: i=1,2 ; j=2,3\} \cup\{\{(1,1),(1,2)\},\{(3,1),(3,2)\}\} \\
& \cup\{\{(i, 1),(l, 3)\}: i=2,3 ; l=1,3\} \cup\{\{(1,1),(1,3)\},\{(2,1),(2,3)\}\} \\
& \cup\{\{(j, 2),(l, 3)\}: j=2,3 ; l=1,2\} \cup\{\{(1,2),(1,3)\},\{(3,2),(3,3)\}\} \\
& \cup\{\{(1,1),(j, 2),(1,3)\}: j=1,2,3\} \cup\{\{(2,1),(2,2),(l, 3)\}: l=1,2\} \\
& \cup\{\{(2,1),(3,2),(l, 3)\}: l=1,2,3\} \cup\{\{(3,1),(3,2),(l, 3)\}: l=1,3\} .
\end{aligned}
$$

Then, by Lemma [5.2, we have the following two subcases:

If $\{(1,1),(1,2)\} \notin \mathcal{S}_{\mathcal{N}}$, then, $\mathcal{S}_{\mathcal{N}} \subseteq \mathcal{S} \backslash\{(1,1),(1,2)\}$ and by Theorem 2.5, one can check that

$$
\left(\left(\begin{array}{ccc}
\frac{8}{14} & \frac{7}{14} & \frac{-1}{14} \\
\frac{3}{14} & \frac{-5}{14} & \frac{2}{14} \\
\frac{3}{14} & \frac{-2}{14} & \frac{-1}{14}
\end{array}\right),\left(\begin{array}{ccc}
\frac{-3}{14} & \frac{7}{14} & \frac{-4}{14} \\
\frac{3}{14} & \frac{7}{14} & \frac{4}{14} \\
0 & 0 & 0
\end{array}\right),\left(\begin{array}{ccc}
\frac{-3}{14} & 0 & \frac{3}{14} \\
0 & \frac{-2}{14} & \frac{2}{14} \\
\frac{3}{14} & \frac{2}{14} & \frac{9}{14}
\end{array}\right)\right)
$$

is a linear solution.

If $\{(1,1),(1,3)\} \notin \mathcal{S}_{\mathcal{N}}$, then $\mathcal{S}_{\mathcal{N}} \subseteq \mathcal{S} \backslash\{\{(1,1),(j, 2),(1,3)\}: j=2,3\}$ and by Theorem 2.5, one can check that

$$
\left(\left(\begin{array}{ccc}
\frac{6}{12} & \frac{3}{12} & \frac{3}{12} \\
\frac{3}{12} & \frac{-3}{12} & 0 \\
\frac{3}{12} & 0 & \frac{-3}{12}
\end{array}\right),\left(\begin{array}{ccc}
\frac{-3}{12} & \frac{4}{12} & \frac{-1}{12} \\
\frac{3}{12} & \frac{7}{12} & \frac{2}{12} \\
0 & \frac{1}{12} & \frac{-1}{12}
\end{array}\right),\left(\begin{array}{ccc}
\frac{-3}{12} & \frac{1}{12} & \frac{2}{12} \\
0 & \frac{-2}{12} & \frac{2}{12} \\
\frac{3}{12} & \frac{1}{12} & \frac{8}{12}
\end{array}\right)\right)
$$


is a linear solution, which proves the theorem for Case 2.2).

For Case 2.3), without loss of generality, we assume $i=1, j=2$ and $l=3$. It can be readily verified that $1 \in m_{1,2}^{2} \cap m_{1,3}^{1} \cap m_{2,3}^{2} ; 2 \in m_{1,3}^{3} \cap m_{2,3}^{3}$ and $3 \in m_{1,2}^{1}$. By Lemma 5.1.

$$
\begin{aligned}
\mathcal{S}_{\mathcal{N}} \subseteq \mathcal{S}: & =\{\{(i, j)\}: 1 \leq i, j \leq 3\} \\
& \cup\{\{(i, 1),(j, 2)\}: i=1,2 ; j=2,3\} \cup\{\{(1,1),(1,2)\},\{(3,1),(3,2)\}\} \\
& \cup\{\{(i, 1),(l, 3)\}: i=2,3 ; l=1,3\} \cup\{\{(1,1),(1,3)\},\{(2,1),(2,3)\}\} \\
& \cup\{\{(j, 2),(l, 3)\}: j=2,3 ; l=1,3\} \cup\{\{(1,2),(1,3)\},\{(2,2),(2,3)\}\} \\
& \cup\{\{(1,1),(j, 2),(1,3)\}: j=1,2,3\} \cup\{\{(2,1),(2,2),(l, 3)\}: l=1,2,3\} \\
& \cup\{\{(2,1),(3,2),(l, 3)\}: l=1,3\} \cup\{\{(3,1),(3,2),(l, 3)\}: l=1,3\} .
\end{aligned}
$$

By Lemma 5.2, we have the following two subcases:

If $\{(1,1),(1,2)\} \notin \mathcal{S}_{\mathcal{N}}$, then, $\mathcal{S}_{\mathcal{N}} \subseteq \mathcal{S} \backslash\{(1,1),(1,2)\}$ and by Theorem 2.5, one can check that

$$
\left(\left(\begin{array}{ccc}
\frac{4}{8} & \frac{3}{8} & \frac{1}{8} \\
\frac{2}{8} & \frac{-2}{8} & 0 \\
\frac{2}{8} & \frac{-1}{8} & \frac{-1}{8}
\end{array}\right),\left(\begin{array}{ccc}
\frac{-2}{8} & \frac{3}{8} & \frac{-1}{8} \\
\frac{2}{8} & \frac{4}{8} & \frac{2}{8} \\
0 & \frac{1}{8} & \frac{-1}{8}
\end{array}\right),\left(\begin{array}{ccc}
\frac{-2}{8} & 0 & \frac{2}{8} \\
0 & \frac{-2}{8} & \frac{2}{8} \\
\frac{2}{8} & \frac{2}{8} & \frac{4}{8}
\end{array}\right)\right)
$$

is a linear solution.

If $\{(1,1),(1,3)\} \notin \mathcal{S}_{\mathcal{N}}$, then, $\mathcal{S}_{\mathcal{N}} \subseteq \mathcal{S} \backslash\{\{(1,1),(1,3)\}\} \backslash\{\{(1,1),(j, 2),(1,3)\}: j=2,3\}$ and by Theorem 2.5, one can check that

$$
\left(\left(\begin{array}{ccc}
\frac{4}{6} & \frac{1}{6} & \frac{1}{6} \\
\frac{1}{6} & \frac{-1}{6} & 0 \\
\frac{1}{6} & 0 & \frac{-1}{6}
\end{array}\right),\left(\begin{array}{ccc}
\frac{-2}{6} & \frac{3}{6} & \frac{-1}{6} \\
\frac{2}{6} & \frac{2}{6} & \frac{2}{6} \\
0 & \frac{1}{6} & \frac{-1}{6}
\end{array}\right),\left(\begin{array}{ccc}
0 & 0 & 0 \\
\frac{-1}{6} & \frac{-1}{6} & \frac{2}{6} \\
\frac{1}{6} & \frac{1}{6} & \frac{4}{6}
\end{array}\right)\right)
$$

is a linear solution, which proves the theorem for Case 2.3).

For Case 2.4), if one of $\mathcal{N}_{t_{1}, t_{2}}, \mathcal{N}_{t_{1}, t_{3}}$ and $\mathcal{N}_{t_{2}, t_{3}}$ is degenerated, then $\mathcal{N}$ has a linear solution by previous cases. So, we assume all of them are non-degenerated and without loss of generality $i=1, j=2$ and $l=3$. Hence, $m_{1,2}^{1}=3, m_{1,2}^{2}=1 ; m_{1,3}^{1}=3, m_{1,3}^{3}=2$; and $m_{2,3}^{2}=1, m_{2,3}^{3}=2$. In the following, consider $s \in \mathcal{S}_{\mathcal{N}} \subseteq \mathcal{S}_{3}$ such that $\alpha(s)=3$. Let $s=\left\{\left(l_{1}, 1\right),\left(l_{2}, 2\right),\left(l_{3}, 3\right)\right\}$. If $l_{1}=3$, then since $m_{1,3}^{1}=3$, we have $l_{3}=3$; if $l_{2}=1$, then since $m_{1,2}^{2}=1$, we have $l_{1}=1$; if $l_{3}=2$, then since $m_{2,3}^{3}=2$, we have $l_{2}=2$. Hence, $\gamma(s)=0$ only if $s=\{(2,1),(3,2),(1,3)\}$, which however, is impossible by Theorem 4.9.

Hence, by Lemma 5.3 ,

$$
\left(\left(\begin{array}{ccc}
\frac{1}{2} & \frac{1}{4} & \frac{1}{4} \\
\frac{1}{4} & \frac{-1}{4} & 0 \\
\frac{1}{4} & 0 & \frac{-1}{4}
\end{array}\right),\left(\begin{array}{ccc}
\frac{-1}{4} & \frac{1}{4} & 0 \\
\frac{1}{4} & \frac{1}{2} & \frac{1}{4} \\
0 & \frac{1}{4} & \frac{-1}{4}
\end{array}\right),\left(\begin{array}{ccc}
\frac{-1}{4} & 0 & \frac{1}{4} \\
0 & \frac{-1}{4} & \frac{1}{4} \\
\frac{1}{4} & \frac{1}{4} & \frac{1}{2}
\end{array}\right)\right) .
$$

is a linear routing solution of $\mathcal{N}$, which completes the proof.

\section{Conclusions and Future Work}

We have settled in this work the Langberg-Médard multiple unicast conjecture for stable 3-pair networks. The conjecture in more general settings, e.g., unstable 3-pair networks and even more general $k$-pair networks, is currently under investigation. 


\section{References}

[1] K. Cai and G. Han, "On network coding advantage for multiple unicast networks," in Proc. ISIT, 2015.

[2] K. Cai and G. Han, "Coding advantage in communications among peers," in Proc. ISIT, 2016.

[3] K. Cai and G. Han, "On the Langberg-Médard multiple unicast conjecture," Journal of Combinatorial Optimization, vol. 34, no. 4, pp. 1114-1132, 2017.

[4] K. Cai and G. Han, "On the Langberg-Médard $k$-unicast conjecture with $k=3,4$," in Proc. ISIT, 2018.

[5] Thomas H. Cormen, Charles E. Leiserson, Ronald L. Rivest, and Clifford Stein, "Introduction to algorithms," MIT Press, Cambridge, MA, third edition, 2009.

[6] G. Han, "Menger's paths with minimum mergings," in Proc. ITW, 2009.

[7] N. Harvey, R. Kleinberg and A. Lehman, "On the capacity of information networks," IEEE Trans. Inf. Theory, vol. 52, no. 6, pp. 2345-2364, Jun. 2006.

[8] K. Jain, V. V. Vazirani and G. Yuval, "On the capacity of multiple unicast sessions in undirected graphs," IEEE/ACM Trans. Networking, vol. 14, pp. 2805-2809, Jun. 2006.

[9] M. Langberg, A. Sprintson and J. Bruck, "The encoding complexity of network coding," IEEE Trans. Inf. Theory, vol. 52, no. 6, pp. 2386-2397, Jun. 2006.

[10] M. Langberg and M. Médard, "On the multiple unicast network coding conjecture," in Proc. 47th Annual Allerton, 2009.

[11] Z. Li and B. Li, "Network coding: The case of multiple unicast sessions," in Proc. 42nd Annual Allerton, 2004.

[12] Z. Li and C. Wu, "Space information flow: multiple unicast," in Proc. ISIT 2012, July $1-6,2012$.

[13] A. Schrijver, "Combinatorial Optimization," Springer-Verlag, 2003.

[14] T. Xiahou, C. Wu, J. Huang and Z. Li, "A geometric framework for investigating the multiple unicast network coding conjecture," in Proc. NetCod, 2012.

[15] Y. Yang, X. Yin, X. Chen, Y. Yang and Z. Li, "A note on the multiple-unicast network coding conjecture," IEEE Communications Letters, vol. 18, no. 5, pp. 869-872, May 2014.

[16] R. Yeung, S.-Y. Li, and N. Cai, Network Coding Theory (Foundations and Trends in Communications and Information Theory), Now Publishers Inc., Hanover, MA, USA, 2006. 